\title{
The effects of cropping systems on soil erosion risks and crop productivity using ImpelERO model and GIS in northeast of Iran
}

\author{
Ehsan Afshar $^{1}$ - Mehrdad Yarnia ${ }^{1}$ - Ali Bagherzadeh ${ }^{2} \cdot$ Reza Sadrabadi Haghighi $^{2}$. \\ Bahram Mirshekari ${ }^{1}$
}

Received: 16 May 2016/Accepted: 22 May 2016/Published online: 6 September 2016

(C) Springer International Publishing Switzerland 2016

\begin{abstract}
Soil erosion is a severe problem worldwide, and controlling it will be a major challenge in the future. Neural networks, as an artificial intelligence technology, have grown rapidly over the past few years and have an ability to deal with nonlinear multivariate systems. An integrated Model to Predict European Land use named ImpelERO is a decision trees/neural network hybrid model. The overall approach of ImpelERO was applied in 49 selected sites from Mashhad-Chenaran plain to quantify the soil erosion features including soil vulnerability index, soil loss rate, erosion risk class and soil depth reduction by sugar beet cultivation under conventional and conservational management practices. Our results revealed that the soil vulnerability indexes ranged from 0.12 to 0.54 and 0.1 to 0.44 by conventional and conservational practices, respectively. The values of soil losses in the study area varied between 4 to $59.7 \mathrm{tha}^{-1}$ year and 3.4 to $38.7 \mathrm{t} \mathrm{ha}^{-1}$ year with an average of 11.72 and $7.83 \mathrm{t} \mathrm{ha}^{-1}$ year by conventional and conservation management practices, respectively. The mean values of erosion risk classes ranged from V3 in conventional to $\mathrm{V} 2$ in conservational practices which categorize the region as accepted tolerable to sensitive to erosion. The long term soil productivity reduction for time horizons 2020, 2050 and 2100 revealed that the conservational practices have greater contribution on preventing productivity reduction than conventional management practices.
\end{abstract}

Mehrdad Yarnia

m.yarnia@yahoo.com

1 Department of Agronomy, Faculty of Agriculture, Tabriz Branch, Islamic Azad University, Tabriz, Iran

2 Department of Agriculture, Mashhad Branch, Islamic Azad University, Mashhad, Iran
Keywords Vulnerability index · Soil loss · Erosion · ImplEro model · Productivity · Sugar beet · GIS

\section{Introduction}

Soil erosion, defined as the detachment and displacement of soil particles from the surface to another location (Govers et al. 1990; Flanagan 2002), continues to be a primary cause of soil degradation throughout the world (Fu and Gulinck 1994), and has become an issue of significant and severe societal and environmental concern (Elsen et al. 2003; Singha et al. 2006). The main causes of soil erosion are inappropriate agricultural practices, deforestation, overgrazing, land abandonment, forest fires and construction activities (Grimm et al. 2002; Yassoglou et al. 1998). Conservation tillage, i.e. the use of tillage techniques which seek to reduce soil disturbance and to maximize soil cover by residues, is primarily used as a means to protect soils from erosion and compaction or to conserve soil moisture. Other reasons for introduction of conservation techniques, e.g. improvement of soil quality and reduction of erosion and top soil loss (López et al. 2000; Machado et al. 2007; Cantero-Martinez et al. 2007; Fuentes et al. 2009) or higher soil moisture and efficiency of water usage (Verch et al. 2009). Conservation tillage, i.e. any form of (reduced) tillage that specifically intends to reduce soil disturbance during seedbed preparation with the objective to maintain soil cover, to improve soil structure and stability while maintaining a viable crop is known to be an effective tool to reduce erosion (Uri et al. 1998; Sisti et al. 2004). Several studies have shown that conservation tillage can significantly reduce erosion: a recent overview of results can be found in Montgomery (2007). However, the degree of reduction in erosion reduction that is reported 
varies significantly from study to study. Numerous studies showed that no tillage practices, with crop residues left on the soil surface increase soil organic carbon (SOC), improve soil aggregation, and preserve the nutrients for plant and soil microorganisms (Hendrix et al. 1998; Jacobs et al. 2009). Conservation tillage is intrinsically linked to residue management, i.e. full, partial or stubble-only retention. Thus, the net effect of any conservation tillage system depends on the integration of the system itself in relation to crop rotation and residue management. Indeed, surface residues accumulated due to conservation tillage were shown to promote higher soil organic $\mathrm{C}$, microbial biomass $\mathrm{N}$ and $\mathrm{C}$, potential $\mathrm{N}$ mineralization and total $\mathrm{N}$ (Salinas-Garcia et al. 2002). Conventional tillage practices including post-harvest residue removal and moldboard ploughing operations accelerate soil organic matter (SOM) oxidation, disrupt soil aggregate stability, and increase soil erosion due to frequent soil disturbance (Frey et al. 1999; Govaerts et al. 2006). Intensive tillage management has caused a significant loss of SOM and serious soil degradation (Liu et al. 2010). Reduced tillage leads to changes in many of the soil's physical properties. Soil bulk density often increases in the $0.1-0.2 \mathrm{~m}$ soil layer in the absence of plouhing. This leads to reduced air-filled pore space and higher water contents. With appropriate crop management, the long-term yields achieved from NT/RT can be at least as high as those achieved from conventionally tilled soils (Grandy et al. 2006; Soane et al. 2012). The soil is more susceptible to water erosion between the beginning of autumn and the end of spring, either because it is unprotected (before sowing) or only partially protected (before the crop reaches full canopy coverage). Therefore, a notillage system, in which crop residues are left on the ground surface, has been proposed to control water erosion (Unger et al. 1991). Residue retention in no tillage systems also favours infiltration (Baumhardt and Lascan 1996; Govaerts et al. 2007). Land use and soil cover are considered as the most important factors affecting the intensity and frequency of runoff and surface erosion (García-Ruiz 2010; Kosmas et al. 1997; Mitchell 1990???). The Middle East region is particularly fragile due to soil and climatic constraints (Kassam 1981). Drought is a constant feature of the region, with considerable annual and inter-annual variation in rainfall. Hence, farming systems have evolved to cope with drought such as wheat (Triticum aestivum) as dominant crop in cultivation (Cooper et al. 1987). Over the last few decades conservation tillage systems, such as shallow cultivation, reduced- or no-till, have been adopted extensively in Iran. This was largely due to labor and energy savings, reduced soil erosion and better soil moisture conservation (Blevins and Frye 1993; Derpsch et al. 2010). The aim of the present study was to assess the effects of cropping management systems including conventional and conservation practices on the values of soil losses, soil vulnerability, erosion risk class and the depth of soil reduction by sugar beet cultivation using neural network based ImpelERO model and GIS in Mashhad plain, northeast of Iran.

\section{Materials and methods}

\section{General characteristics of the study area}

The present study was conducted in Mashhad plain with an area of $6,131 \mathrm{~km}^{2}$, Khorasan-e-Razavi province, northeast Iran (Fig. 1). The study area is located between latitude $35^{\circ} 59^{\prime}$ to $37^{\circ} 04^{\prime} \mathrm{N}$ and longitude $58^{\circ} 22^{\prime}$ to $60^{\circ} 07^{\prime} \mathrm{E}$ including lands less than $1500 \mathrm{~m}$ above sea level (asl). The topographical elevation values of the study area vary between 900 and $1500 \mathrm{~m}$ asl, while the main topographical elevation range over $1200 \mathrm{~m}$ asl. The general physiographic trend of the plain extends in a NW-SE direction with an average of $160 \mathrm{~km}$ length surrounded between two mountainous zones of Kopetdagh at northward and Binaloud at southward based on visual interpretation of satellite image and field observations (Fig. 2).

Geologically, main alluvial nature of the plain has been developed into a thick sediment dominated environment belongs to quaternary period. The main land use practice at the study area is irrigated farming around Kashaf-rud River. The study area has a semi-arid climate with mean annual precipitation of $222.1 \mathrm{~mm}$ and mean annual temperature of $15.8{ }^{\circ} \mathrm{C}$. The rainiest month is March $(44.8 \mathrm{~mm})$ and the driest month is September $(1.2 \mathrm{~mm})$. The soil profiles dataset including 49 selected sites were used for the study, where each selected site represents a land unit. Dataset files on climate data for meteorological stations closer to the selected sites including monthly means of temperature and precipitation correspond to 1981-2010 period were compiled from the Iran Meteorological Organization. The soil physical and chemical characteristics and the land terrain of the selected sites have been presented in Tables 1 and 2. The following cropping systems were considered on the selected application sites: Sugar beet under conventional practice and conservational practice. Table 3 summarizes for each cropping system, the agronomic practices which were assumed for all the sites and used as input data in the ImpelERO model application. The baseline and estimated yields were used to derive the productivity level. The tillage implements sequence, along with the number of times applied and the workability consideration by the farmer, were also established. These management practices, with special reference to the tillage operations, are frequently applied for sugar beet in irrigated condition. 
Fig. 1 Location and geographical position of the study area
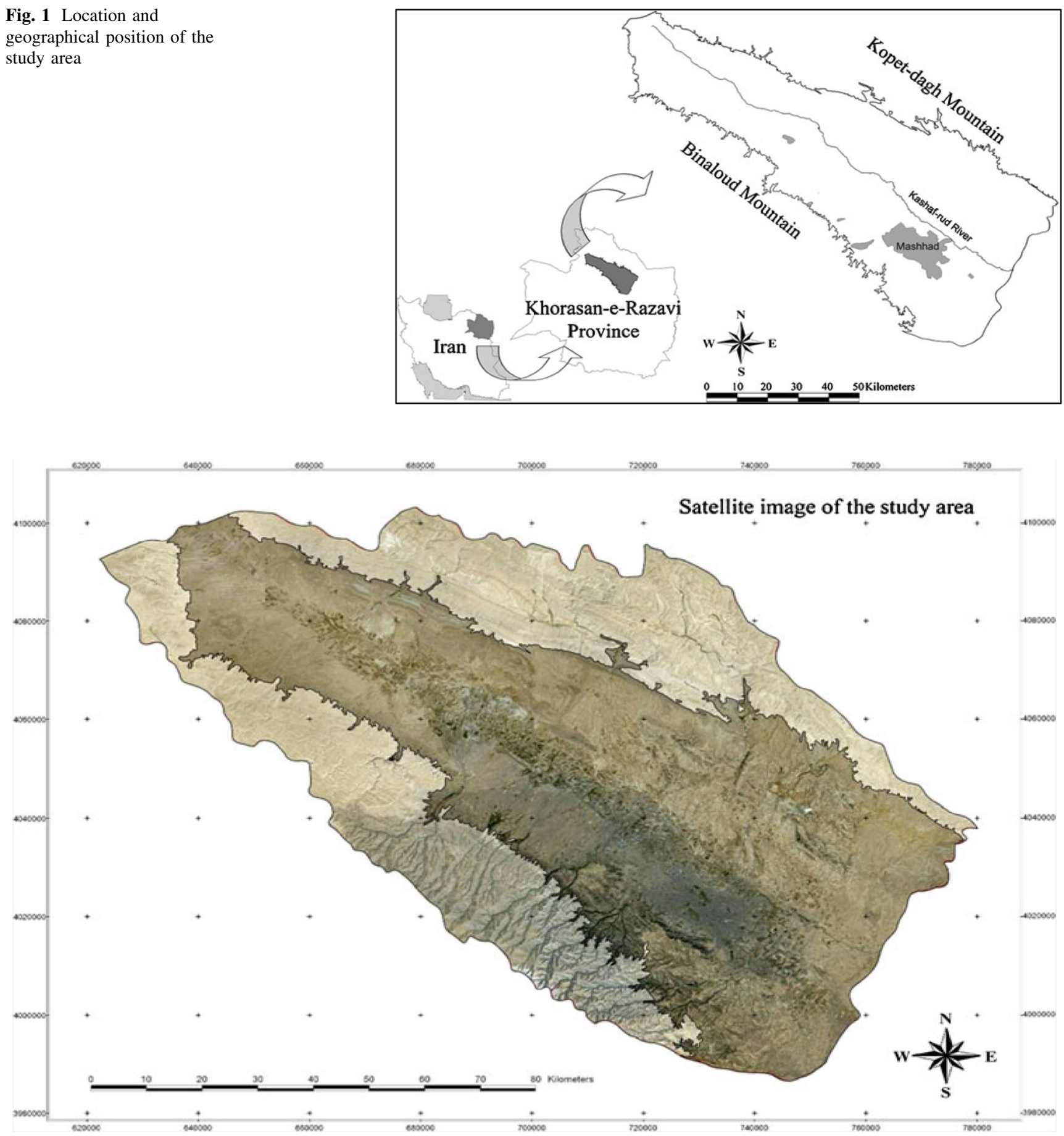

Fig. 2 Satellite image of the study area

\section{Soil erosion estimation}

Soil erosion parameters were calculated on the basis of the expert system/neural networks structure of the ImpelERO model (De la Rosa et al. 1999). This model was developed as a Universal Soil Loss Equation-type model following traditional land evaluation analysis and advanced empirical modeling techniques. Using expert-decision trees, soil survey information and expert knowledge of the soil erosion process were combined with land and management qualities. An artificial neural networks approach was then applied to capture the interactions between the land and different management practices for winter sugar beet production in order to predict the vulnerability index to soil 
Table 1 Soil physical and chemical characteristics of the study area

\begin{tabular}{|c|c|c|c|c|c|c|c|c|c|}
\hline Site & Longitude & Latitude & Sand & Silt & Clay & Texture & $\operatorname{ESP}(\%)$ & $\mathrm{OM}(\%)$ & Bulk density \\
\hline 1 & 59.745 & 36.111 & 32 & 50 & 18 & Silt loam/loam & 14.1 & 1.07 & 1.407 \\
\hline 2 & 59.683 & 36.214 & 39 & 45 & 16 & Loam & 4.41 & 1.26 & 1.438 \\
\hline 3 & 59.757 & 36.147 & 21 & 57 & 22 & Silt loam & 10.5 & 1.1 & 1.357 \\
\hline 4 & 59.684 & 36.349 & 14 & 56 & 30 & Silty clay loam & 5.76 & 1.34 & 1.298 \\
\hline 5 & 59.835 & 36.144 & 45 & 41 & 14 & Loam & 21.8 & 0.52 & 1.469 \\
\hline 6 & 59.751 & 36.208 & 17 & 61 & 22 & Silt loam & 5.09 & 0.88 & 1.349 \\
\hline 7 & 59.839 & 36.207 & 25 & 64 & 11 & Silt loam & 2.04 & 0.67 & 1.466 \\
\hline 8 & 59.843 & 36.270 & 39 & 33 & 28 & Clay loam & 10.5 & 0.95 & 1.356 \\
\hline 9 & 59.770 & 36.336 & 33 & 41 & 26 & Loam & 5.63 & 0.76 & 1.355 \\
\hline 10 & 59.608 & 36.350 & 32 & 54 & 14 & Silt loam & 2.18 & 0.88 & 1.444 \\
\hline 11 & 59.618 & 36.406 & 41 & 47 & 12 & Loam & 0.58 & 0.67 & 1.484 \\
\hline 12 & 59.699 & 36.400 & 39 & 49 & 12 & Loam & 0.43 & 0.47 & 1.48 \\
\hline 13 & 59.540 & 36.410 & 34 & 52 & 14 & Silt loam & 5.49 & 1.03 & 1.448 \\
\hline 14 & 59.466 & 36.476 & 33 & 53 & 14 & Silt loam & 4.96 & 0.59 & 1.446 \\
\hline 15 & 59.544 & 36.473 & 37 & 49 & 14 & Loam & 0.88 & 0.48 & 1.454 \\
\hline 16 & 59.629 & 36.470 & 41 & 40 & 19 & Loam & 2.61 & 0.6 & 1.416 \\
\hline 17 & 59.700 & 36.466 & 33 & 47 & 20 & Loam & 0.73 & 0.48 & 1.394 \\
\hline 18 & 59.392 & 36.482 & 54 & 34 & 12 & Sandy loam & 2.75 & 0.72 & 1.509 \\
\hline 19 & 59.215 & 36.506 & 64 & 26 & 10 & Sandy loam & 1.46 & 0.78 & 1.555 \\
\hline 20 & 59.316 & 36.553 & 15 & 53 & 32 & Silty clay loam & 4.41 & 1.43 & 1.29 \\
\hline 21 & 59.392 & 36.538 & 30 & 49 & 21 & Loam & 3.59 & 1.17 & 1.381 \\
\hline 22 & 59.470 & 36.538 & 43 & 40 & 17 & Loam & 3.73 & 1.17 & 1.437 \\
\hline 23 & 59.549 & 36.535 & 22 & 53 & 25 & Silt loam & 12.8 & 1.02 & 1.34 \\
\hline 24 & 59.627 & 36.532 & 30 & 51 & 19 & Silt loam & 2.18 & 0.84 & 1.395 \\
\hline 25 & 59.236 & 36.597 & 24 & 50 & 26 & Silt loam/loam & 3.31 & 2.34 & 1.338 \\
\hline 26 & 59.318 & 36.608 & 26 & 59 & 15 & Silt loam & 10.1 & 0.93 & 1.422 \\
\hline 27 & 59.396 & 36.605 & 32 & 47 & 21 & Loam & 1.75 & 0.48 & 1.385 \\
\hline 28 & 59.475 & 36.602 & 33 & 47 & 20 & Loam & 1.31 & 0.22 & 1.394 \\
\hline 29 & 59.084 & 36.618 & 32 & 55 & 13 & Silt loam & 2.18 & 1.03 & 1.455 \\
\hline 30 & 59.010 & 36.684 & 26 & 62 & 12 & Silt loam & 0.88 & 0.81 & 1.455 \\
\hline 31 & 59.088 & 36.681 & 28 & 50 & 22 & Silt loam/loam & 3.45 & 2.78 & 1.37 \\
\hline 32 & 59.166 & 36.677 & 24 & 58 & 18 & Silt loam & 3.17 & 1.6 & 1.392 \\
\hline 33 & 59.322 & 36.671 & 25 & 51 & 24 & Silt loam & 0.58 & 1 & 1.352 \\
\hline 34 & 59.394 & 36.670 & 32 & 51 & 17 & Silt loam & 0.88 & 0.52 & 1.416 \\
\hline 35 & 58.935 & 36.749 & 14 & 58 & 28 & Silty clay loam & 6.02 & 1.91 & 1.308 \\
\hline 36 & 59.013 & 36.746 & 36 & 60 & 4 & Silt loam & 7.71 & 1.09 & 1.636 \\
\hline 37 & 59.092 & 36.743 & 44 & 31 & 25 & Loam & 59.8 & 0.91 & 1.382 \\
\hline 38 & 59.170 & 36.740 & 58 & 31 & 11 & Sandy loam & 0.58 & 0.41 & 1.53 \\
\hline 39 & 58.782 & 36.818 & 34 & 48 & 18 & Loam & 0.88 & 0.62 & 1.411 \\
\hline 40 & 58.861 & 36.815 & 26 & 48 & 26 & Loam & 12.2 & 0.72 & 1.342 \\
\hline 41 & 58.939 & 36.812 & 25 & 48 & 27 & Clay loam/loam & 8.59 & 1.03 & 1.334 \\
\hline 42 & 58.707 & 36.884 & 34 & 56 & 10 & Silt loam & 0.58 & 0.93 & 1.497 \\
\hline 43 & 58.786 & 36.881 & 29 & 49 & 22 & Loam & 13.3 & 0.59 & 1.372 \\
\hline 44 & 58.554 & 36.953 & 32 & 52 & 16 & Silt loam & 0.43 & 0.86 & 1.425 \\
\hline 45 & 58.633 & 36.950 & 48 & 40 & 12 & Loam & 0.58 & 0.88 & 1.498 \\
\hline 46 & 58.711 & 36.947 & 25 & 52 & 23 & Silt loam & 6.42 & 1.84 & 1.358 \\
\hline 47 & 58.558 & 37.016 & 32 & 54 & 14 & Silt loam & 0.43 & 1 & 1.444 \\
\hline 48 & 58.636 & 37.013 & 30 & 52 & 18 & Silt loam & 0.58 & 0.59 & 1.403 \\
\hline 49 & 58.697 & 37.012 & 24 & 58 & 18 & Silt loam & 0.29 & 0.76 & 1.392 \\
\hline
\end{tabular}


Table 2 Land terrain values of the study area

\begin{tabular}{|c|c|c|c|c|c|}
\hline \multirow[t]{2}{*}{ Site } & \multicolumn{2}{|c|}{ Slope } & \multirow[t]{2}{*}{ Aspect degree } & \multirow[t]{2}{*}{ Sub soil stoniness class ${ }^{\mathrm{b}}$} & \multirow[t]{2}{*}{ Internal drainage $^{c}$} \\
\hline & $\%$ & Class $^{a}$ & & & \\
\hline 1 & 1.00 & $\mathrm{~F}$ & 3.00 & $\mathrm{C}$ & M \\
\hline 2 & 1.00 & $\mathrm{~F}$ & 3.00 & $\mathrm{C}$ & M \\
\hline 3 & 1.00 & $\mathrm{~F}$ & 3.00 & $\mathrm{C}$ & M \\
\hline 4 & 2.00 & A & 7.00 & $\mathrm{~F}$ & $\mathrm{~V}$ \\
\hline 5 & 2.00 & A & 3.00 & $\mathrm{C}$ & $\mathrm{M}$ \\
\hline 6 & 1.00 & $\mathrm{~F}$ & 3.00 & $\mathrm{C}$ & M \\
\hline 7 & 4.00 & G & 3.00 & $\mathrm{~F}$ & M \\
\hline 8 & 1.00 & $\mathrm{~F}$ & 7.00 & $\mathrm{~F}$ & $\mathrm{~V}$ \\
\hline 9 & 1.00 & $\mathrm{~F}$ & 7.00 & $\mathrm{~F}$ & M \\
\hline 10 & 4.00 & G & 3.00 & $\mathrm{~F}$ & M \\
\hline 11 & 2.00 & A & 7.00 & $\mathrm{~F}$ & M \\
\hline 12 & 4.00 & G & 5.00 & $\mathrm{~F}$ & M \\
\hline 13 & 2.00 & A & 3.00 & $\mathrm{~F}$ & $\mathrm{M}$ \\
\hline 14 & 2.00 & A & 3.00 & $\mathrm{~F}$ & $\mathrm{M}$ \\
\hline 15 & 2.00 & A & 7.00 & $\mathrm{~F}$ & $\mathrm{M}$ \\
\hline 16 & 1.00 & $\mathrm{~F}$ & 5.00 & $\mathrm{~F}$ & $\mathrm{M}$ \\
\hline 17 & 2.00 & A & 7.00 & $\mathrm{~F}$ & $\mathrm{M}$ \\
\hline 18 & 2.00 & A & 3.00 & $\mathrm{~F}$ & $\mathrm{H}$ \\
\hline 19 & 2.00 & A & 3.00 & $\mathrm{C}$ & $\mathrm{H}$ \\
\hline 20 & 2.00 & A & 3.00 & $\mathrm{~F}$ & $\mathrm{~V}$ \\
\hline 21 & 2.00 & A & 3.00 & $\mathrm{~F}$ & $\mathrm{M}$ \\
\hline 22 & 5.00 & $\mathrm{G}$ & 5.00 & $\mathrm{~F}$ & $\mathrm{M}$ \\
\hline 23 & 1.00 & $\mathrm{~F}$ & 7.00 & $\mathrm{~F}$ & $\mathrm{M}$ \\
\hline 24 & 2.00 & A & 7.00 & $\mathrm{~F}$ & $\mathrm{M}$ \\
\hline 25 & 1.00 & $\mathrm{~F}$ & 3.00 & $\mathrm{C}$ & $\mathrm{M}$ \\
\hline 26 & 1.00 & $\mathrm{~F}$ & 7.00 & $\mathrm{~F}$ & $\mathrm{M}$ \\
\hline 27 & 2.00 & A & 7.00 & $\mathrm{~F}$ & $\mathrm{M}$ \\
\hline 28 & 1.00 & $\mathrm{~F}$ & 7.00 & $\mathrm{~F}$ & $\mathrm{M}$ \\
\hline 29 & 2.00 & A & 3.00 & $\mathrm{C}$ & $\mathrm{M}$ \\
\hline 30 & 2.00 & A & 3.00 & $\mathrm{C}$ & $\mathrm{M}$ \\
\hline 31 & 1.00 & $\mathrm{~F}$ & 3.00 & $\mathrm{C}$ & $\mathrm{M}$ \\
\hline 32 & 1.00 & $\mathrm{~F}$ & 3.00 & $\mathrm{~F}$ & $\mathrm{M}$ \\
\hline 33 & 2.00 & A & 7.00 & $\mathrm{~F}$ & $\mathrm{M}$ \\
\hline 34 & 2.00 & A & 7.00 & $\mathrm{~F}$ & $\mathrm{M}$ \\
\hline 35 & 1.00 & $\mathrm{~F}$ & 3.00 & $\mathrm{C}$ & $\mathrm{V}$ \\
\hline 36 & 2.00 & A & 3.00 & $\mathrm{C}$ & $\mathrm{M}$ \\
\hline 37 & 2.00 & A & 7.00 & $\mathrm{~F}$ & $\mathrm{M}$ \\
\hline 38 & 2.00 & A & 7.00 & $\mathrm{~F}$ & $\mathrm{H}$ \\
\hline 39 & 1.00 & $\mathrm{~F}$ & 5.00 & $\mathrm{C}$ & $\mathrm{M}$ \\
\hline 40 & 1.00 & $\mathrm{~F}$ & 3.00 & $\mathrm{~F}$ & $\mathrm{M}$ \\
\hline 41 & 2.00 & A & 7.00 & $\mathrm{~F}$ & $\mathrm{~V}$ \\
\hline 42 & 1.00 & $\mathrm{~F}$ & 3.00 & $\mathrm{~F}$ & $\mathrm{M}$ \\
\hline 43 & 1.00 & $\mathrm{~F}$ & 5.00 & $\mathrm{~F}$ & $\mathrm{M}$ \\
\hline 44 & 2.00 & A & 3.00 & $\mathrm{~F}$ & $\mathrm{M}$ \\
\hline 45 & 2.00 & A & 3.00 & $\mathrm{~F}$ & M \\
\hline 46 & 2.00 & A & 7.00 & $\mathrm{~F}$ & $\mathrm{M}$ \\
\hline 47 & 2.00 & A & 3.00 & $\mathrm{~F}$ & $\mathrm{M}$ \\
\hline 48 & 2.00 & A & 7.00 & $\mathrm{~F}$ & $\mathrm{M}$ \\
\hline 49 & 7.00 & $\mathrm{U}$ & 7.00 & $\mathrm{~F}$ & M \\
\hline
\end{tabular}

a Slope class: $F$ flat, $A$ almost flat, $G$ gently undul, $U$ undul

b Sub soil stoniness class: $F$ few, $C$ common

c Internal drainage: $H$ rapid, $M$ moderate, $V$ very slow 
Table 3 Agricultural management practices for sugar beet cultivation, and assumed to be used in the selected sites

\begin{tabular}{|c|c|c|c|c|c|c|c|c|c|}
\hline \multirow[t]{2}{*}{ Crop type } & \multirow[t]{2}{*}{ Management } & \multirow{2}{*}{$\begin{array}{l}\text { Residue } \\
\text { treatment }\end{array}$} & \multirow{2}{*}{$\begin{array}{l}\text { Tillage } \\
\text { system }\end{array}$} & \multirow{2}{*}{$\begin{array}{l}\text { Row } \\
\text { spacing } \\
(\mathrm{m})\end{array}$} & \multirow{2}{*}{$\begin{array}{l}\text { Baseline } \\
\text { yield } \\
\left.(\mathrm{t} \mathrm{ha})^{-1}\right)^{\mathrm{a}}\end{array}$} & \multirow{2}{*}{$\begin{array}{l}\text { Estimated } \\
\text { yield } \\
\left(\mathrm{t} \mathrm{ha}^{-1}\right)^{\mathrm{b}}\end{array}$} & \multicolumn{3}{|l|}{ Tillage operation } \\
\hline & & & & & & & Implement & Time $^{c}$ & Workability $^{\mathrm{d}}$ \\
\hline \multirow[t]{7}{*}{ Spring crop } & \multirow[t]{7}{*}{ Convention } & \multirow[t]{7}{*}{ Grazing } & \multirow[t]{7}{*}{ Traditional } & \multirow[t]{7}{*}{0.6} & \multirow[t]{7}{*}{36} & \multirow[t]{7}{*}{38.5} & Plow Moldboard & 2 & Yes \\
\hline & & & & & & & Drill deep furrow & 1 & Yes \\
\hline & & & & & & & Disc cultivator & 3 & Yes \\
\hline & & & & & & & Harrow-roller & 4 & Yes \\
\hline & & & & & & & Planter row & 1 & Yes \\
\hline & & & & & & & Spray implement & 4 & Yes \\
\hline & & & & & & & Fertilizer applicator & 3 & Yes \\
\hline \multirow[t]{6}{*}{ Spring crop } & \multirow[t]{6}{*}{ Conservation } & \multirow[t]{6}{*}{ Nil } & \multirow[t]{6}{*}{ Conservation } & \multirow[t]{6}{*}{0.6} & \multirow[t]{6}{*}{40.5} & \multirow[t]{6}{*}{42} & Drill conventional, no till & 1 & Yes \\
\hline & & & & & & & Disc cultivator & 2 & Yes \\
\hline & & & & & & & Harrow-roller & 2 & Yes \\
\hline & & & & & & & Planter row & 1 & Yes \\
\hline & & & & & & & Spray implement & 4 & Yes \\
\hline & & & & & & & Fertilizer applicator & 3 & Yes \\
\hline
\end{tabular}

a Baseline yield is the actual value of crop production from statistical sources

b Estimated yield is the predicted value of crop production by using simulation models

c Number of times that an implement is used

d Workability status makes reference if (yes or no) the optimum soil water content for each tillage operation is considered by the farmer

erosion, erosion risk class, soil loss rate and soil depth reduction.

\section{Results and discussion}

\section{Soil vulnerability Index}

The values of soil vulnerability indexes ranged from 0.12 to 0.54 by conventional and 0.1 to 0.44 by conservation management practices for sugar beet cultivation. It was revealed that the index values of soil vulnerability by conventional managements in the study area were higher than the corresponding values by conservation management practices (Table 4). Undulated slopes, southward aspects, fine and/or silty soil texture and low drainage classes were found the most important factors affecting soil vulnerability index by conventional management practices; however, the effects of these parameters was reduced to $22.3 \%$ in average by shifting to conservation management practices. The most vulnerable soils to erosion were found in some areas at southeast, middle and northwest of the plain, while the effect of conservation practices by reduced tillage and using soil stabilizers restricted the vulnerable areas to small parts in the middle and northwest of the study area (Fig. 3). García-Orenes et al. (2009) found that straw mulch was able to significantly improve soil properties after a 16 months study period in a Mediterranean farming area. Mulumba and Lal (2008) found that straw application as soil stabilizer increased total porosity and soil aggregation with increasing mulching rates resulted in lower vulnerability to soil erosion. Similar positive relationships between organic matter and aggregate stability indices were found by García-Orenes et al. (2005) and Zornoza et al. (2007).

\section{Soil loss estimation}

The values of soil losses in the study area varied between 4 to $59.7 \mathrm{t} \mathrm{ha}^{-1}$ year and 3.4 to $38.7 \mathrm{t} \mathrm{ha}^{-1}$ year with an average of 11.72 and $7.83 \mathrm{t} \mathrm{ha}^{-1}$ year by conventional and conservation management practices, respectively. The mean values of erosion risk classes ranged from V3 in conventional to V2 in conservational practices which categorize the region as accepted tolerable to sensitive to erosion (Table 4; Fig. 6). As expected the values of soil losses by conventional practices of sugar beet cultivation were higher than the corresponding values by conservation management practices (Table 4). According to the Technical Working Group on Erosion as part of European Union Soil Thematic Strategy (Jones et al. 2004) an average annual soil loss of more than $1 \mathrm{t} \mathrm{ha}^{-1}$ can be considered as irreversible within a time span of 50-100 years. When the definition considers the maximum permissible rate at which soil fertility can be maintained, tolerable rates have a much larger range, $1 \mathrm{t} \mathrm{ha}^{-1}$ year (Schaub and Prasuhn 1998) to as much as 15-20 $\mathrm{t} \mathrm{ha}^{-1}$ year where soils are deeper (Schertz 1983). A mean annual 
Table 4 Soil erosion parameters by conventional and conservational practices for sugar beet cultivation

\begin{tabular}{|c|c|c|c|c|c|c|c|c|}
\hline \multicolumn{5}{|c|}{ Conventional practices } & \multicolumn{4}{|c|}{ Conservational practices } \\
\hline Site & $\begin{array}{l}\text { Vulnerability } \\
\text { index } \\
(0.00-1.00)\end{array}$ & $\begin{array}{l}\text { Risk class } \\
\text { (V1-V5) }\end{array}$ & $\begin{array}{l}\text { Soil loss rate } \\
\left(\mathrm{t} \mathrm{ha}^{-1} \text { year }^{-1}\right)\end{array}$ & $\begin{array}{l}\text { Soil depth } \\
\text { reduction } \\
\left(\mathrm{cm} \text { year }^{-1}\right)\end{array}$ & $\begin{array}{l}\text { Vulnerability } \\
\text { index } \\
(0.00-1.00)\end{array}$ & $\begin{array}{l}\text { Risk class } \\
\text { (V1-V5) }\end{array}$ & $\begin{array}{l}\text { Soil loss rate } \\
\left(\mathrm{t} \mathrm{ha}^{-1} \text { year }^{-1}\right)\end{array}$ & $\begin{array}{l}\text { Soil depth } \\
\text { reduction } \\
\left(\mathrm{cm} \mathrm{year}^{-1}\right)\end{array}$ \\
\hline 1 & 0.26 & $\mathrm{~V} 2$ & 8.7 & 0.06 & 0.2 & $\mathrm{~V} 2$ & 6.6 & 0.05 \\
\hline 2 & 0.22 & $\mathrm{~V} 2$ & 7.3 & 0.05 & 0.18 & $\mathrm{~V} 2$ & 5.9 & 0.04 \\
\hline 3 & 0.26 & $\mathrm{~V} 2$ & 8.7 & 0.06 & 0.2 & $\mathrm{~V} 2$ & 6.6 & 0.05 \\
\hline 4 & 0.38 & V3 & 25.1 & 0.19 & 0.3 & $\mathrm{~V} 2$ & 9.8 & 0.08 \\
\hline 5 & 0.26 & $\mathrm{~V} 2$ & 8.7 & 0.06 & 0.2 & $\mathrm{~V} 2$ & 6.6 & 0.05 \\
\hline 6 & 0.26 & $\mathrm{~V} 2$ & 8.7 & 0.06 & 0.2 & $\mathrm{~V} 2$ & 6.6 & 0.05 \\
\hline 7 & 0.26 & $\mathrm{~V} 2$ & 8.7 & 0.06 & 0.2 & $\mathrm{~V} 2$ & 6.6 & 0.05 \\
\hline 8 & 0.43 & V3 & 36.5 & 0.27 & 0.34 & $\mathrm{~V} 3$ & 17.2 & 0.13 \\
\hline 9 & 0.26 & $\mathrm{~V} 2$ & 8.7 & 0.06 & 0.2 & V2 & 6.6 & 0.05 \\
\hline 10 & 0.26 & V2 & 8.7 & 0.06 & 0.2 & V2 & 6.6 & 0.05 \\
\hline 11 & 0.26 & V2 & 8.7 & 0.06 & 0.2 & V2 & 6.6 & 0.04 \\
\hline 12 & 0.26 & V2 & 8.7 & 0.06 & 0.2 & V2 & 6.6 & 0.04 \\
\hline 13 & 0.26 & V2 & 8.7 & 0.06 & 0.2 & V2 & 6.6 & 0.05 \\
\hline 14 & 0.26 & V2 & 8.7 & 0.06 & 0.2 & V2 & 6.6 & 0.05 \\
\hline 15 & 0.26 & V2 & 8.7 & 0.06 & 0.2 & V2 & 6.6 & 0.05 \\
\hline 16 & 0.26 & V2 & 8.7 & 0.06 & 0.2 & V2 & 6.6 & 0.05 \\
\hline 17 & 0.26 & V2 & 8.7 & 0.06 & 0.2 & V2 & 6.6 & 0.05 \\
\hline 18 & 0.3 & V3 & 10.7 & 0.07 & 0.26 & V2 & 8.7 & 0.06 \\
\hline 19 & 0.3 & V3 & 10.7 & 0.07 & 0.26 & V2 & 8.7 & 0.06 \\
\hline 20 & 0.38 & V3 & 25.1 & 0.19 & 0.3 & V2 & 9.8 & 0.08 \\
\hline 21 & 0.26 & V2 & 8.7 & 0.06 & 0.2 & V2 & 6.6 & 0.05 \\
\hline 22 & 0.26 & V2 & 8.7 & 0.06 & 0.2 & V2 & 6.6 & 0.05 \\
\hline 23 & 0.26 & V2 & 8.7 & 0.07 & 0.2 & $\mathrm{~V} 2$ & 6.6 & 0.05 \\
\hline 24 & 0.26 & V2 & 8.7 & 0.06 & 0.2 & V2 & 6.6 & 0.05 \\
\hline 25 & 0.22 & V2 & 7.3 & 0.05 & 0.18 & V2 & 5.9 & 0.04 \\
\hline 26 & 0.26 & V2 & 8.7 & 0.06 & 0.2 & $\mathrm{~V} 2$ & 6.6 & 0.05 \\
\hline 27 & 0.26 & V2 & 8.7 & 0.06 & 0.2 & $\mathrm{~V} 2$ & 6.6 & 0.05 \\
\hline 28 & 0.26 & V2 & 8.7 & 0.06 & 0.2 & V2 & 6.6 & 0.05 \\
\hline 29 & 0.26 & V2 & 8.7 & 0.06 & 0.2 & $\mathrm{~V} 2$ & 6.6 & 0.05 \\
\hline 30 & 0.26 & V2 & 8.7 & 0.06 & 0.2 & $\mathrm{~V} 2$ & 6.6 & 0.05 \\
\hline 31 & 0.22 & V2 & 7.3 & 0.05 & 0.18 & V2 & 5.9 & 0.04 \\
\hline 32 & 0.22 & V2 & 7.3 & 0.05 & 0.18 & V2 & 5.9 & 0.04 \\
\hline 33 & 0.26 & V2 & 8.7 & 0.06 & 0.2 & V2 & 6.6 & 0.05 \\
\hline 34 & 0.26 & V2 & 8.7 & 0.06 & 0.2 & V2 & 6.6 & 0.05 \\
\hline 35 & 0.38 & V3 & 25.1 & 0.19 & 0.3 & V2 & 9.8 & 0.08 \\
\hline 36 & 0.26 & V2 & 8.7 & 0.05 & 0.2 & V2 & 6.6 & 0.04 \\
\hline 37 & 0.26 & V2 & 8.7 & 0.06 & 0.2 & V2 & 6.6 & 0.05 \\
\hline 38 & 0.12 & V1 & 4 & 0.03 & 0.1 & V1 & 3.4 & 0.02 \\
\hline 39 & 0.26 & V2 & 8.7 & 0.06 & 0.2 & V2 & 6.6 & 0.05 \\
\hline 40 & 0.26 & V2 & 8.7 & 0.07 & 0.2 & V2 & 6.6 & 0.05 \\
\hline 41 & 0.43 & V3 & 36.5 & 0.27 & 0.34 & V3 & 17.2 & 0.13 \\
\hline 42 & 0.26 & V2 & 8.7 & 0.06 & 0.2 & V2 & 6.6 & 0.04 \\
\hline 43 & 0.26 & V2 & 8.7 & 0.06 & 0.2 & V2 & 6.6 & 0.05 \\
\hline 44 & 0.26 & V2 & 8.7 & 0.06 & 0.2 & V2 & 6.6 & 0.05 \\
\hline 45 & 0.26 & V2 & 8.7 & 0.06 & 0.2 & V2 & 6.6 & 0.04 \\
\hline
\end{tabular}


Table 4 continued

\begin{tabular}{|c|c|c|c|c|c|c|c|c|}
\hline \multicolumn{5}{|c|}{ Conventional practices } & \multicolumn{4}{|c|}{ Conservational practices } \\
\hline Site & $\begin{array}{l}\text { Vulnerability } \\
\text { index } \\
(0.00-1.00)\end{array}$ & $\begin{array}{l}\text { Risk class } \\
\text { (V1-V5) }\end{array}$ & $\begin{array}{l}\text { Soil loss rate } \\
\left(\mathrm{t} \mathrm{ha}^{-1} \text { year }^{-1}\right)\end{array}$ & $\begin{array}{l}\text { Soil depth } \\
\text { reduction } \\
\left(\mathrm{cm} \mathrm{year}^{-1}\right)\end{array}$ & $\begin{array}{l}\text { Vulnerability } \\
\text { index } \\
(0.00-1.00)\end{array}$ & $\begin{array}{l}\text { Risk class } \\
\text { (V1-V5) }\end{array}$ & $\begin{array}{l}\text { Soil loss rate } \\
\left(\mathrm{t} \mathrm{ha}^{-1} \text { year }^{-1}\right)\end{array}$ & $\begin{array}{l}\text { Soil depth } \\
\text { reduction } \\
\left(\mathrm{cm} \mathrm{year}^{-1}\right)\end{array}$ \\
\hline 46 & 0.22 & $\mathrm{~V} 2$ & 7.3 & 0.05 & 0.18 & $\mathrm{~V} 2$ & 5.9 & 0.04 \\
\hline 47 & 0.26 & $\mathrm{~V} 2$ & 8.7 & 0.06 & 0.2 & $\mathrm{~V} 2$ & 6.6 & 0.05 \\
\hline 48 & 0.26 & $\mathrm{~V} 2$ & 8.7 & 0.06 & 0.2 & $\mathrm{~V} 2$ & 6.6 & 0.05 \\
\hline 49 & 0.54 & V4 & 59.7 & 0.43 & 0.44 & V3 & 38.7 & 0.28 \\
\hline
\end{tabular}

The Zonation of Vulnerability Index Using ImpelERO model for Sugar Beet Cultivation in Mashhad Plain
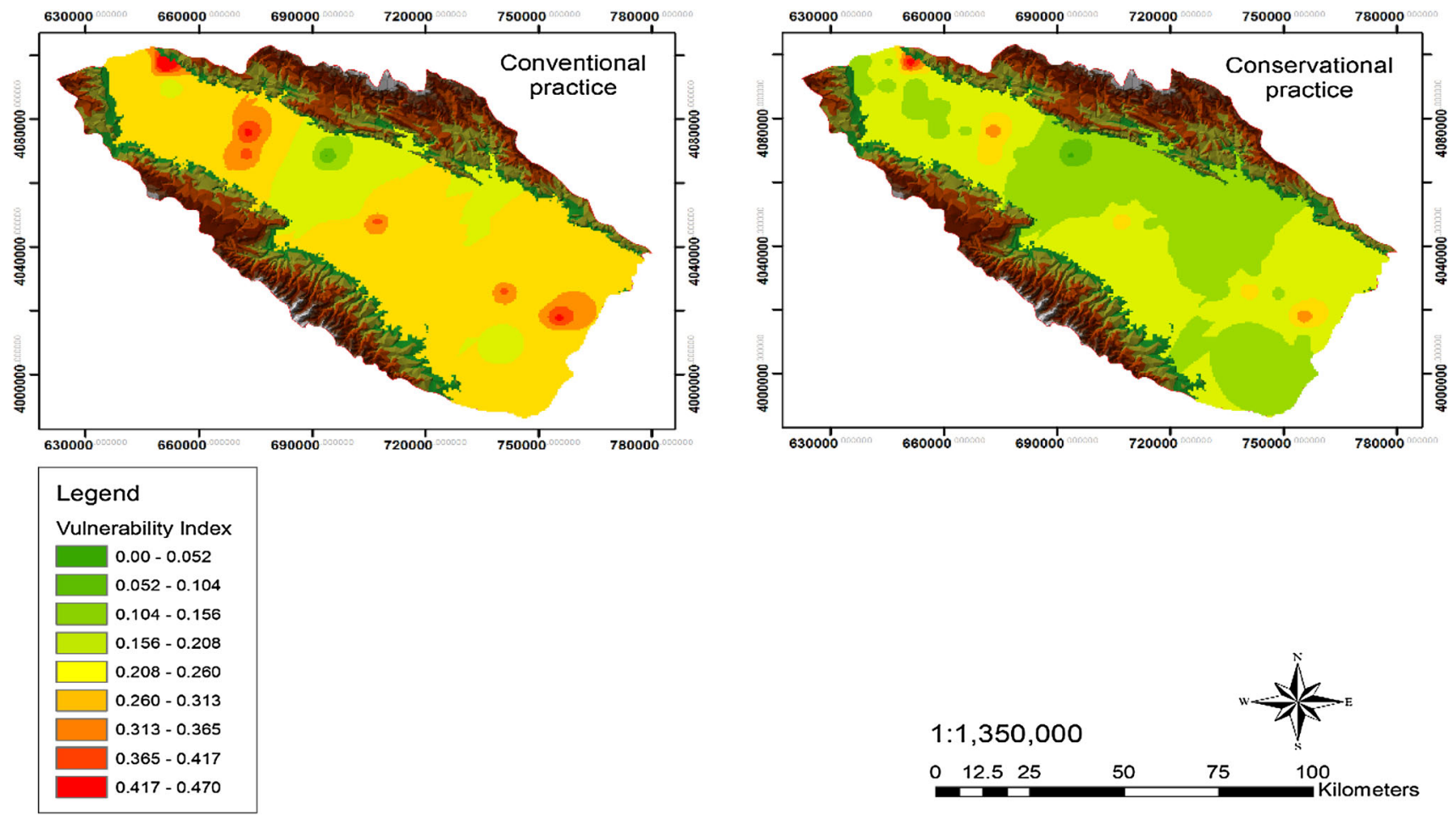

Fig. 3 The zonation of vulnerability index for sugar beet cultivation in Mashhad plain

soil loss of $11 \mathrm{t} \mathrm{ha}^{-1}$ is a widely accepted tolerable soil loss value, but values as low as $2 \mathrm{t} \mathrm{ha}^{-1}$ are recommended for sensitive areas where soils are thin and highly erodible (Hudson 1981). The advantages of conservation tilling and straw residue treatment versus the use of conventional tillage practices and grazing straw treatment in terms of less soil loss have been documented by Raclot et al. (2009). Studies have revealed that in dry areas, approximately $50 \%$ of the biomass from herbaceous vegetation roots is added to the soil as OM (USDA 2000). It has also been noted that covers help to retain OM and nutrients in sloping soil (Gay et al. 2004), which limits crusting due to fracturing of the crusts themselves (Aljibury and Christensen
1972). In compare to conventional tillage practices the average values of soil losses by conservation management practices showed a reduction of $33.2 \%$. Several studies have shown that conservation tillage can significantly reduce erosion (Montgomery 2007). A 6-years study was set up in Belgium to study the effects of conservation tillage on runoff and soil loss under Western European conditions using rainfall simulation. Over 250 rainfall simulation experiments on small plots were carried out on fields with sugar beet, maize and winter wheat. These experiments clearly showed a reduction of inter rill soil loss and runoff on the conservation tillage plots, with a mean reduction of 43 and $23 \%$ with respect to the values 


\section{The Zonation of Soil Loss Rate Using ImpelERO model for Sugar Beet Cultivation in Mashhad Plain}
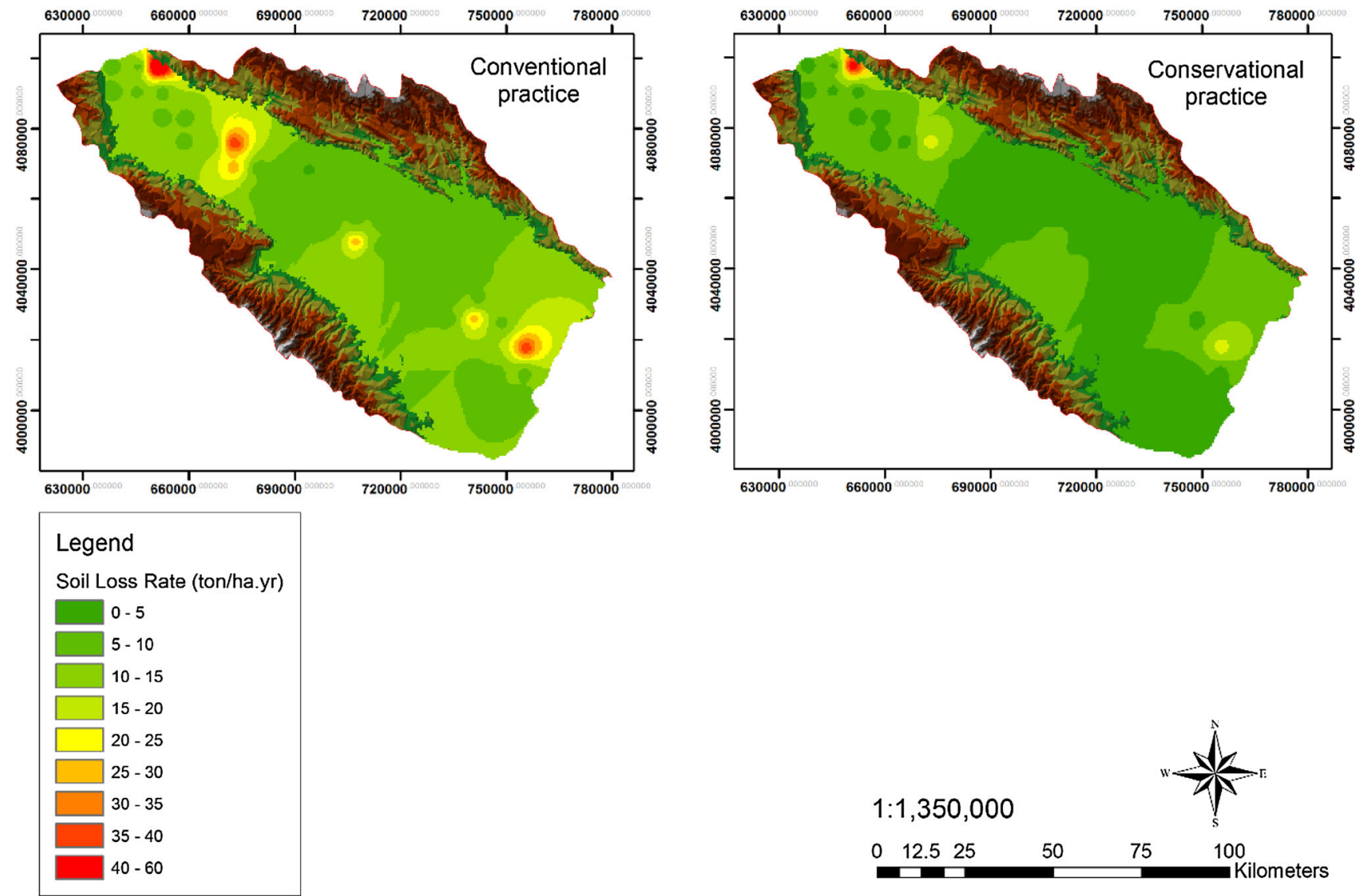

Fig. 4 The zonation of soil loss rate for sugar beet cultivation in Mashhad plain

measured on paired, conventional tillage plots, respectively (Leys et al. 2007). Corresponds to vulnerable areas of the plain, the zonation of soil losses by conventional management practices revealed that the most soil loss values are scattered in southeast and northwest of the plain, where the slopes are more undulated and southward, soil texture is fine and/or silty, the drainage is very slow and the soil organic matter is commonly low (Fig. 4).

\section{Long-term changes in crop productivity}

Following soil loss prediction, the rate of change in relative productivity of soils can be calculated on the basis that favorable rooting characteristics are present in the soil profile. As erosion removes the upper soil profile, productivity will decline if the subsoil is limiting for crop growth. The effect of diminishing soil organic matter and nutrient contents on crop productivity is not considered in this analysis. The concept of soil loss tolerance was introduced in order to give a measurement of how much erosion a soil could tolerate before experiencing excessive damage. In general terms, the soil loss tolerance is defined as the maximum rate of annual soil erosion that will permit a high level of crop productivity to be sustainable. This tolerance value is considered to range from 1 to $10 \mathrm{Mg} \mathrm{ha}^{-1}$ per year depending upon intrinsic soil profile characteristics (Larson 1981). According to the profile characteristics of the dominant soils of the study area the soils were grouped as maximum tolerance (Soil group A), which corresponds to deep alluvial soils with favorable characteristics to a depth exceeding $120 \mathrm{~cm}$. Soil erosion at the rate estimated by the ImpelERO model was simulated for four time horizons: current, 2020, 2050 and 2100. Vulnerability index, soil loss and soil depth reduction rates are facilitated directly by the model application; and the corresponding soil depth reductions are calculated using the depth reduction rate (Table 4; Fig. 5). After calculations of soil erosion, crop productivity was calculated at each time horizon by application of the MicroLEISALBERO sugar beet yield prediction model (De la Rosa 1996) (Fig. 6). The predicted yield (PY) was converted to productivity index $(\mathrm{Pi})$ as follows: 
The Zonation of Soil Depth Reduction Using ImpelERO model for Sugar Beet Cultivation in Mashhad Plain
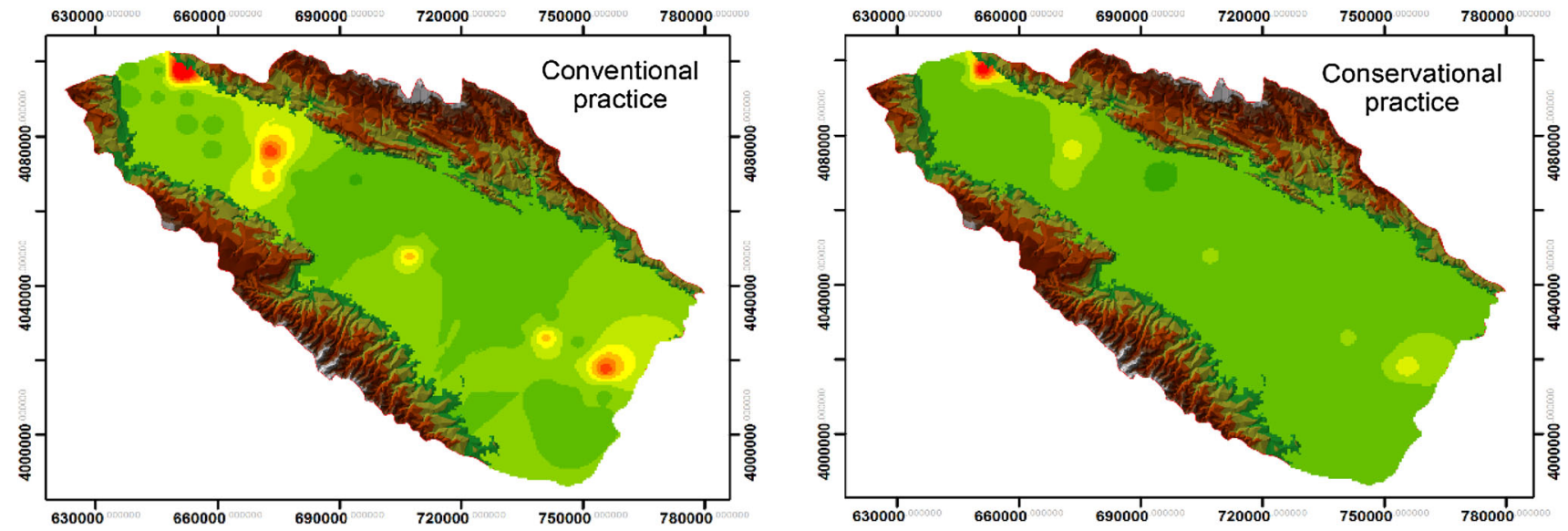

\begin{tabular}{|l}
\hline Legend \\
Soil Depth Reduction (cm/yr) \\
$0.000-0.035$ \\
$0.035-0.070$ \\
$0.070-0.105$ \\
$0.105-0.140$ \\
$0.140-0.175$ \\
$0.175-0.210$ \\
$0.210-0.245$ \\
$0.245-0.280$ \\
$0.280-0.318$ \\
\hline$\square$
\end{tabular}

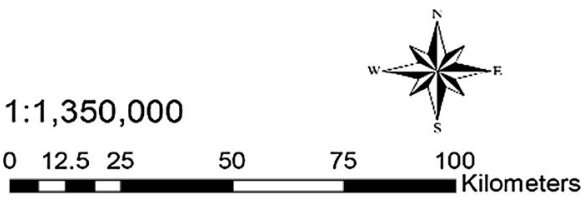

Fig. 5 The zonation of soil depth reduction for sugar beet cultivation in Mashhad plain

The Zonation of Erosion Risk Class Using ImpelERO model for Sugar Beet Cultivation in Mashhad Plain
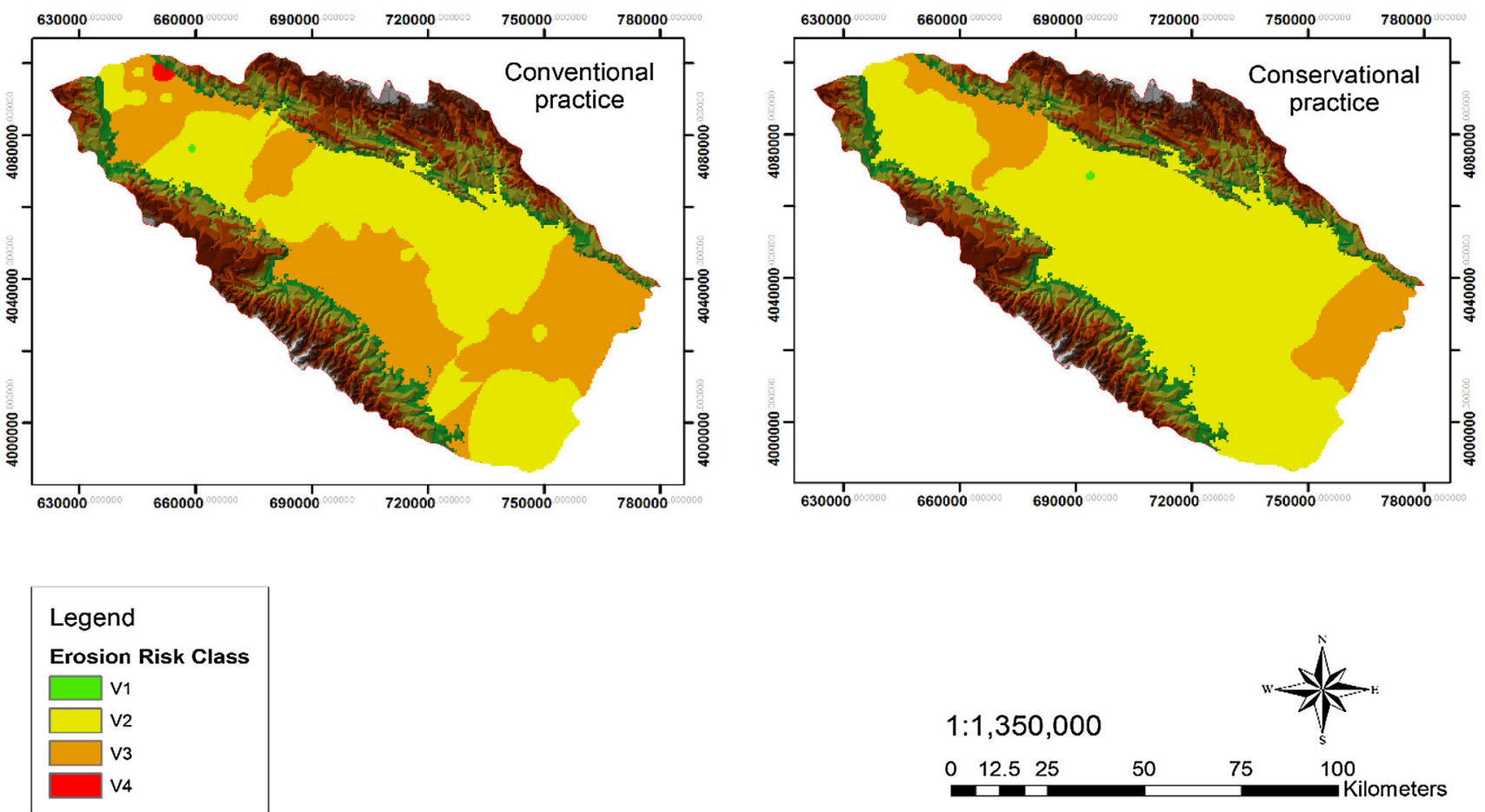

Fig. 6 The zonation of erosion risk class for sugar beet cultivation in Mashhad plain 


\section{$\mathrm{Pi}=\mathrm{PY} / \max \mathrm{PY}$}

where max (PY) was considered to be $43.2 \mathrm{t} \mathrm{ha}^{-1}$. By using this procedure for the four time horizons, the rooting depth moved down the profile as soils eroded, unless some limiting layer occurred in the first $120 \mathrm{~cm}$ or until a limiting layer was encountered. Figures 7 and 8 show productivity changes with three simulated time horizons: 2020, 2050 and 2100 for the 49 sites. For the land unit with high vulnerability index an initial productivity index of 0.81 was calculated. While the current erosion rate in this land unit ranged from 59.7 to $38.7 \mathrm{t} \mathrm{ha}^{-1}$ year $^{-1}$ by conventional and conservation practices, the productivity index under these practices dropped to 0.745 to 0.772 , revealed a loss of 8 and $4.7 \%$, for the time horizon 2100 of simulated erosion, respectively. For 5 land units with moderately medium vulnerability index the average productivity index of 0.91 was estimated. As the average current soil loss rate in these land units ranged from 29.66 to $12.76 \mathrm{t} \mathrm{ha}^{-1}$ year $^{-1}$ by conventional and conservation practices, the productivity index under these practices dropped to 0.875 to 0.898 , exhibited a loss of 3.86 and $1.36 \%$, for the time horizon 2100 of simulated erosion,
Fig. 7 Productivity changes with the time horizon for the 49 sites under conventional practice in Mashhad plain

Fig. 8 Productivity changes with the time horizon for the 49 sites under conservational practice in Mashhad plain

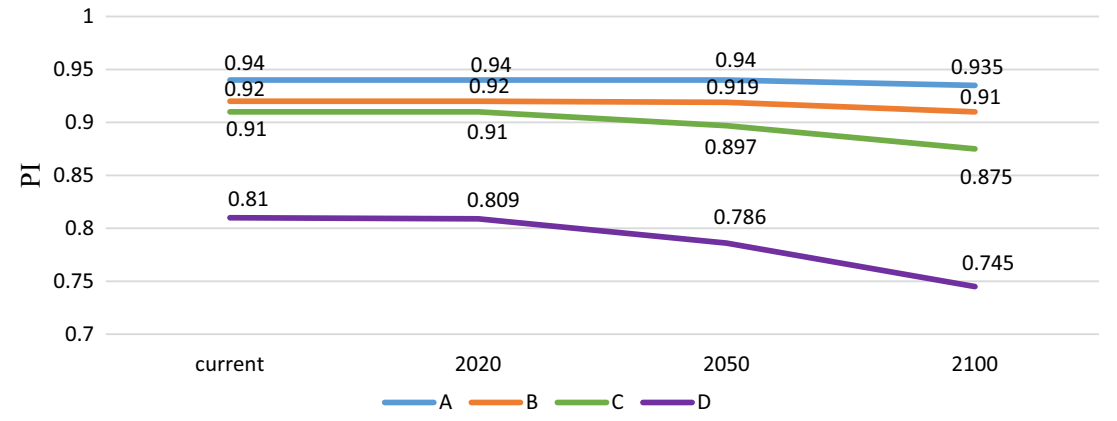

A: The Productivity Index for very low soil vulnerability class

B: The Productivity Index for low soil vulnerability class

C: The Productivity Index for moderate soil vulnerability class

D: The Productivity Index for high soil vulnerability class

A: The Productivity Index for very low soil vulnerability class

B: The Productivity Index for low soil vulnerability class

C: The Productivity Index for moderate soil vulnerability class

D: The Productivity Index for high soil vulnerability class

Table 5 The regression equation between long term productivity reductions versus soil depth reduction

\begin{tabular}{lcllllll}
\hline Vulnerability index & Number of sites & \multicolumn{2}{l}{ Conventional practice } & & \multicolumn{2}{l}{ Conservational practice } \\
& & & Regression equation & $\mathrm{R}^{2}$ & & Regression equation & $\mathrm{R}^{2}$ \\
\hline High & 1 & - & - & - & - \\
Moderate & 5 & $\mathrm{y}=0.0421 \mathrm{x}+0.0595$ & 0.99 & $\mathrm{y}=0.0544 \mathrm{x}+0.026$ & 0.98 \\
Low & 37 & $\mathrm{y}=0.0222 \mathrm{x}+0.0389$ & 0.52 & $\mathrm{y}=0.0192 \mathrm{x}+0.0415$ & 0.5 \\
Very low & 6 & $\mathrm{y}=0.0204 \mathrm{x}+0.0361$ & 0.51 & $\mathrm{y}=0.0667 \mathrm{x}+0.02$ & 0.99 \\
\hline
\end{tabular}


respectively. For 37 land units with low vulnerability index the mean productivity index of 0.92 were calculated. While the average current soil loss rate in these land units ranged from 8.81 to $6.71 \mathrm{t} \mathrm{ha}^{-1}$ year $^{-1}$ by conventional and conservation practices, the productivity index under these practices dropped to 0.91 to 0.916 , showed a loss of 0.99 and $0.4 \%$, for the time horizon 2100 of simulated erosion, respectively. For the rest 6 land units with very low vulnerability index the mean productivity index of 0.94 were determined. As the average current soil loss rate in above land units ranged from 6.75 to $5.48 \mathrm{t} \mathrm{ha}^{-1}$ year $^{-1}$ by conventional and conservation practices, the productivity index under these practices dropped to 0.935 to 0.937 , showed a loss of 0.52 and $0.25 \%$, for the time horizon 2100 of simulated erosion, respectively. The regression equation between long term productivity reductions in percentage versus soil depth reduction in centimeter by conventional and conservation management practices indicated a high correlation between these parameters (Table 5).

Our results revealed that although productivity is sustained for a short time, but it will drop with continued erosion in long term period. Comparing two management practices, no special differences were observed on productivity index at medium, low and very low soil vulnerability indexes over long term time horizon. Only by conservation management practice the productivity was less declined compared to conventional practice over long time period (Figs. 7, 8).

Acknowledgments We thank Islamic Azad University, Tabriz Branch for their support of the project. Thanks also to one anonymous reviewer for suggestions on data analyses and interpretation.

\section{References}

Aljibury FK, Christensen LP (1972) Water penetration of vineyard soils as modified by cultural practices. Am J Enol Vitic 23(1):35-38

Baumhardt RL, Lascan RJ (1996) Rain infiltration as affected by wheat residue amount and distribution in ridged tillage. Soil Sci Soc Am J 60:1908-1913

Blevins RL, Frye WW (1993) Conservation tillage:an ecological approach to soil management. Adv Agron 51:33-78

Cantero-Martinez C, Angas P, Lampurlanes J (2007) Long-term yield and water use efficiency under various tillage systems in Mediterranean rainfed conditions. Ann Appl Biol 150:293-305

Cooper PJM, Gregory PJ, Tully D, Harris H (1987) Improving wateruse efficiency of annual crops in the rain-fed farming system of West Asia and North Africa. Exp Agric 23:113-158

De la Rosa D (1996) MicroLEIS 4.1: microcomputer based land evaluation information system. Integrated System for Land Data transfer and Agro-ecological Land Evaluation. Software + Documentation. CSIC-IRNAS Publication, Sevilla. http://www. irnase.csic.es/microlei.htm

De la Rosa D, Mayol F, Moreno JA, Bonsón T, Lozano S (1999) An expert-system/neural-network model (ImpelERO) for evaluating agricultural soil erosion in Andalucia region, southern of Spain. Agric Ecosyst Environ 73:211-226

Derpsch R, Friedrich T, Kassam A, Hongwen L (2010) Current status of adoption of no-till farming in the world and some of its main benefits. Int J Agric Biol Eng 3:1-25

Elsen E, Hessel R, Liu B (2003) Discharge and sediment measurements at the outlet of a watershed on the Loess plateau of China. Catena 54:147-160

Flanagan D (2002) In: Lal R (ed) Erosion encyclopedia of soil science. Marcel Dekker, New York, pp 395-398

Frey SD, Elliott ET, Paustian K (1999) Bacterial and fungal abundance and biomass in conventional and no-tillage agroecosystems along two climatic gradients. Soil Biol Biochem 31:573-585

Fu B, Gulinck H (1994) Land evaluation in an area of severe erosion: the Loess Plateau of China. Land Degrad Dev 5:33-40

Fuentes M, Govaerts B, De León F, Hidalgo C, Dendooven L, Sayre KD, Etchevers J (2009) Fourteen years of applying zero and conventional tillage, crop rotation and residue management systems and its effect on physical and chemical soil quality. Eur J Agron 30:228-237

García-Orenes F, Guerrero C, Mataix-Solera J, Navarro-Pedreño J, Gómez I, Mataix- Beneyto J (2005) Factors controlling the aggregate stability and bulk density in two different degraded soils amended with biosolids. Soil Tillage Res 82:65-76

García-Orenes F, Cerdà A, Mataix-Solera J, Guerrero C, Bodí MB, Arcenegui V, Zornoza R, Sempere JG (2009) Effects agricultural management on surface soil properties and soil-waterlosses in eastern Spain. Soil Tillage Res 106:117-123

García-Ruiz JM (2010) The effects of land uses on soil erosion in Spain: a review. Catena 81:1-11

Gay G, Bovio M, Minati JL, Morando A, Novello V, Ambrosoli R (2004) Soil management in relation to training system in a steep vineyard. J Int Sci Vigne Vin 38(1):71-74

Govaerts B, Mezzalama M, Sayre KD, Crossa J, Nicol JM, Deckers J (2006) Longterm consequences of tillage, residue management, and crop rotation on maize/wheat root rot and nematode populations in subtropical highlands. Appl Soil Ecol 32:305-315

Govaerts B, Sayre KD, Lichter K, Dendooven L, Deckers J (2007) Influence of permanent raised bed planting and residue management on physical and chemical soil quality in rain fed maize/ wheat systems. Plant Soil 291:39-54

Govers G, Everaert W, Poesen J, Rauws G, De Ploey J, Latridou JP (1990) A long flume study of the dynamic factors affecting the resistance of a loamy soil to concentrated flow erosion. Earth Surf Proc Land 11:515-524

Grandy AS, Robertson GP, Thelen KD (2006) Do productivity and environmental trade-offs justify periodically cultivating no-till cropping systems? Agron J 98(1377):1383

Grimm M, Jones R, Montanarella L (2002) Soil erosion risk in Europe. European Commission, Institute for Environment and Sustainability, European Soil Bureau, Ispra

Hendrix PF, Franzluebbersm AJ, McCracken DV (1998) Management effects on carbon accumulation and loss in soils on the southern Appalachian Piedmont of Georgia, USA. Soil Tillage Res $47: 245-251$

Hudson NW (1981) Soil conservation, 2nd edn. Batsford, London

Jacobs A, Rauber R, Ludwing B (2009) Impact of reduced tillage on carbon and nitrogen storage of two Haplic Luvisols after 40 years. Soil Tillage Res 102:158-164

Jones RJA, Le Bissonnais Y, Bazzoffi P, Sanchez Diaz J, Düwel O, Loj G, Øygarden L, Prasuhn V, Rydell B, Strauss P, Berenyi Uveges J, Vandekerckhove L, Yordanov Y (2004) Nature and extent of soil erosion in Europe. In: Van-Camp L, Bujarrabal B, Gentile AR, Jones RJA, Montanarella L, Olazabal C, Selvaradjou S-K (eds), Reports of the technical working groups 
established under the thematic strategy for soil protection. Volume II Erosion. EUR 21319 EN/2. Office for Official Publications of the European Communities, Luxembourg, pp $145-185$

Kassam A (1981) Climate, soil and land resources in North Africa and West Asia. Plant Soil 58:1-29

Kosmas C, Danalatos N, Cammeraat LH, Chabart M, Diamantopoulos J, Farand R (1997) The effect of land use on runoff and soil erosion rates under Mediterranean conditions. Catena 29:45-59

Larson WE (1981) Predicting the soil resource base. J Soil Water Conserv 36:13-16

Leys A, Govers G, Gillijns K, Poesen J (2007) Conservation tillage on loamy soils: explaining the variability in interrill runoff and erosion reduction. Eur J Soil Sci 58(6):1425-1436

Liu XB, Herbert SJ, Zhang XY (2010) Feeding China's growing needs for grain. Nature 465:420

López MV, Gracia R, Arrúe JL (2000) Effects of reduced tillage on soil surface properties affecting wind erosion in semiarid lands of Central Aragó n. Eur J Agron 12:191-199

Machado S, Petrie S, Rhinhart K, Qu A (2007) Long-term continuous cropping in the Pacific Northwest: tillage and fertilizer effects on winter wheat, spring wheat, and spring barley production. Soil Tillage Res 94:473-481

Mitchell DJ (1990) The use of vegetation and land use parameters in modelling catchment sediment yields. In: Thornes JB (ed) Vegetation and erosion, processes and environments. Wiley, Chichester, pp 289-314

Montgomery D (2007) Soil erosion and agricultural sustainability. Proc Natl Acad Sci USA 104(33):13268-13272

Mulumba LN, Lal R (2008) Mulching effects on selected soil physical properties. Soil Tillage Res 98:106-111

Raclot D, Le Bissonnais Y, Louchart X, Andrieux P, Moussa R, Voltz M (2009) Soil tillage and scale effects on erosion from fields to catchment in a Mediterranean vineyard area. Agric Ecosyst Environ 134:201-210

Salinas-Garcia JR, Velasquez-Garcia J, De J, Gallerdo-Valdez M, Diaz-Maderos P, Callallero-Henandez F, Topia-Vargas LM,
Rosales-Robles E (2002) Tillage effects on microbial biomass and nutrient distribution in soils under rainfed corn production in central western Mexico. Soil Tillage Res 60:143-152

Schaub D, Prasuhn V (1998) A map on soil erosion on arable land as a planning tool for sustainable land use in Switzerland. In: Blume HP, Eger H, Fleischhauer E, Hebel A, Reij C, Steiner KG (eds), Towards sustainable land use, volumes I and II: furthering cooperation between people and institutions. Advances in Geoecology, pp 161-168

Schertz DL (1983) The basis for soil loss tolerance. J Soil Water Conserv 38:10-14

Singha R, Tiwarib KN, Malb BC (2006) Hydrological studies for small watershed in India using the ANSWERS model. J Hydrol 318:184-199

Sisti CPJ, Santos HP, Kohhann R, Alves BJR, Urquiaga S, Boddey RM (2004) Change in carbon and nitrogen stocks in soil under 13 years of conventional or zero tillage in southern Brazil. Soil Tillage Res 76(1):39-58

Soane BD, Ball BC, Arvidsson J, Basch G, Moreno F, Roger-Estrade $\mathrm{J}$ (2012) No till in northern, western and south-western Europe: a review of problems and opportunities for crop production and the environment. Soil Tillage Res 118:66-87

Unger PW, Stewart BA, Parr JF, Singh RP (1991) Crop residue management and tillage methods for conserving soil and water in semi-arid regions. Soil Tillage Res 20:219-240

Uri N, Atwood J, Sanabria J (1998) The environmental benefits and costs of conservation tillage. Sci Total Environ 216:13-32

USDA (2000) Interpreting indicators of rangeland health, Version 3, 2000, TR: 1734-1736, BLM

Verch G, Kächele H, Höltl K, Richter C, Fuchs C (2009) Comparing the profitability of tillage methods in Northeast Germany: a field trial from 2002 to 2005. Soil Tillage Res 104(1):16-21

Yassoglou N, Montanarella L, Govers G, Van Lynden G, Jones RJA, Zdruli P (1998) Soil erosion in Europe. Technical report for DG XI. European Soil Bureau, Ispra

Zornoza R, Mataix-Solera J, Guerrero C, Arcenegui V, Mayoral AM, Morales J, Mataix-Beneyto J (2007) Soil properties under natural forest in the Alicante Province of Spain. Geoderma 142:334-341 